\title{
Review of: "A Concise Study of Schizophrenia and Resting- state fMRI data analysis"
}

\author{
Ajay Kumar \\ 1 Central University of Himachal Pradesh
}

Potential competing interests: The author(s) declared that no potential competing interests exist.

This paper is written in nice way. It's a very informative for new learner like us. Schizophrenia a major psychiatric disorder, is a devastating illness. So in this context every sentence written in this paper is in very organized way. I like this paper most. 